\section{Response to Nakaguma et al.}

We thank Nakaguma et al. ${ }^{1}$ for their clinical report letter in response to our publication of 12 families with autosomal recessive LZTR1-associated Noonan syndrome. ${ }^{2}$ We were surprised to have identified 12 families with this disorder and expect that there are quite a few more, of which the Nakaguma et al. ${ }^{1}$ report is only the first. In characterizing pleiotropic syndromes, it is always challenging to distinguish coincidence from cause and effect. Uncommon manifestations of rare disorders are challenging from a statistical point of view and so every such report is useful to consider. A second observation of growth hormone deficiency supports (but does not prove) the hypothesis that this is a low frequency, but associated component of the syndrome. This helps improve our understanding of the pleiotropy of LZTR1related Noonan syndrome and alerts clinicians that growth hormone deficiency should be considered in patients with this diagnosis. Their identification of two novel variants in LZTR1 expands the allelic heterogeneity of the disorder and is consistent with our model that the disorder is caused by either biallelic hypomorphic variants or compound heterozygosity for one hypomorphic variant and one loss-of-function variant. As we noted in our publication, we strongly suspect that biallelic loss-of-function variants are nonviable.

We encourage centers who have large cohorts of patients with Noonan syndrome to evaluate unsolved cases for biallelic variants in LZTR1. As well, variants in this gene should be considered in all patients with apparent Noonan syndrome who do not have another explanation for their disorder. Even more importantly, the hypothesis-free approach of exome and genome sequencing of children with other presenting phenotypes is likely to identify patients with biallelic variants in this gene, expanding the phenotype beyond what we currently recognize as Noonan syndrome.

\section{DISCLOSURE}

LGB received support from the Intramural Research Program of the National Human Genome Research Institute grants HG200328-11 and HG200388-03. LGB receives royalties from Genentech Corp, is an advisor to the Illumina Corp, and receives honoraria from Wiley-Blackwell.

Leslie G. Biesecker, $M D^{1}$

${ }^{1}$ National Human Genome Research Institute, National Institutes of Health, Bethesda, MD, USA. Correspondence: Leslie G. Biesecker (lesb@mail.nih.gov)

\section{REFERENCES}

1. Leslie G. Biesecker, Response to Nakaguma et al. Genet Med (this issue); 10.1038/s41436-018-0041-5.

2. Johnston JJ, van der Smagt JJ, Rosenfeld JA, et al. Autosomal recessive Noonan syndrome associated with biallelic LZTR1 variants. Genet Med 2018; doi:10.1038/gim.2017.249.

Advance online publication 30 June 2018. doi:10.1038/s41436-018-0042-4 